\title{
Influence of stress state and strain path on deformation induced martensitic transformations
}

\author{
E.S. Perdahcıoğlu*, H.J.M. Geijselaers, J. Huétink \\ University of Twente, Faculty of Engineering Technology - P.O. Box 217, 7500AE, The Netherlands \\ * Corresponding author: Tel: +31 53 4894175; fax: +3153 4893471; e-mail: e.s.perdahcioglu@ctw.utwente.nl
}

\begin{abstract}
This article investigates the mechanically induced transformation behavior of $12 \mathrm{Cr}-9 \mathrm{Ni}$ 4Mo (ASTM A 564) austenitic stainless steel under different stress states. This phenomenon is studied experimentally on a plane stress biaxial test facility. The facility can load a sheet specimen simultaneously in shear and tension which enables us to investigate the effect of stress state on transformation kinetics. The martensite fraction is monitored via a magnetic sensor while the strain is measured using a camera and a dot-tracking software.

Keywords: Strain induced transformation, biaxial test, stress state, strain path, TRIP
\end{abstract}

\section{Introduction}

Solid-solid phase transformations have been the subject of many studies for years due to their influence on the mechanical properties of materials. Special interest has been focused on metastable materials such as austenitic stainless steels which exploit the martensitic transformations to combine very good mechanical properties such as high formability and strength. These properties are very attractive to the industry especially for automotive and home appliances. A major drawback of these steels however, is the difficulty of predicting processes like sheet metal forming. It is obvious that to have a good constitutive model the physical factors that underly the martensitic transformation must be well understood and implemented.

The experiments done by Angel [1] show that plastic straining induces martensitic transformation in TRIP steels. This strain induced transformation phenomenon has been studied by many authors since then and what emerges from these studies is that the kinetics of the transformation is significantly influenced by factors such as the stress state and temperature. According to Olson \& Cohen [2] the plastic strain drives the transformation by the generation of nucleation sites through shear band intersections [3][4]. The stress on the other hand provides the mechanical driving force needed for the transformation. This driving force can be calculated using the crystallographic theory of martensite formation [5][6] and following the Magee theory the progress of transformation can be modeled [7][8]. These studies clearly pronounce the effect of the state of stress on the transformation. The temperature at which the deformation takes place dictates the chemical driving force which is basically the difference of the Gibbs free energies of the constituent phases. And in various experimental studies the effect of temperature is obtained [2][9][10].

In sheet metal forming processes the local stress state on the material can have any arbitrary combination of shear and tension. Consequently, for the same amount of plastic straining the kinetics of transformation will differ at different points in the material causing unexpected shape changes in the final product. Additionally, a material point in the sheet might undergo strain path changes during the forming operation which will result in a varying stress state at that point. There are a number of studies that focus on the effect of stress state on the transformation kinetics [8][9]. However, the number of experimental studies that can clearly demonstrate the effect is small. Mostly, tests in which different points in the material undergo different deformation paths are considered e.g. singleshear tests [11]. In these studies different stress 
states are imposed simultaneously on different locations in the material [12][13][14] which limits the evaluation of the investigation. In addition, there are multiaxial tests that focus on the transformation plasticity and kinetics during the athermal transformation of martensite $[15][16]$.

In this study the aim is to observe the effect of stress state on the kinetics of strain induced martensitic transformation using a biaxial test facility which can deform sheet material in arbitrary proportions of shear and tension. The martensite fraction is monitored during the deformation process with a magnetic sensor which enables a complete visualization of the transformation process.

\section{$2 \quad$ Experimental setup}

\subsection{Test facility and the sample}

The biaxial test facility is illustrated in figure 1a. Two separate clamps constrain the upper and the lower sections of the sample. The upper clamp can move only horizontally whereas the lower clamp can move only vertically. The horizontal and vertical displacements are controlled independently via separate actuators.

With this setup it is possible to impose a constant stress state on the deformation zone during the test by keeping the direction of the deformation rate constant. This direction is controlled via the relative speed of the clamps as illustrated in figure $1 \mathrm{~b}$, enabling a range of tests from plane strain tension to simple shear.

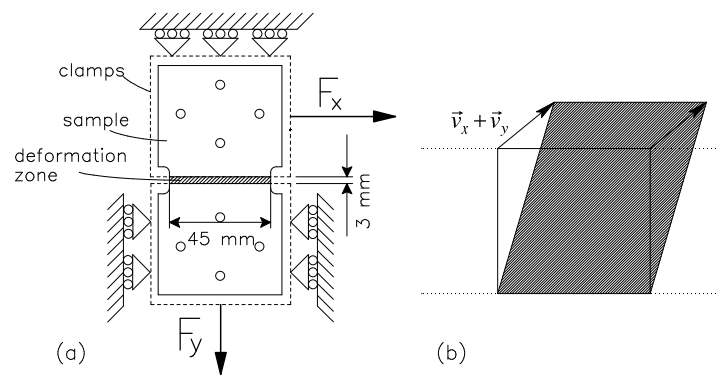

Figure 1: (a) The biaxial test setup and the deformation zone. (b) The stress state is controlled by the horizontal and vertical deformation speeds.

\section{$2.2 \quad$ Stress state and strain}

The dimensions of the deformation zone $(\mathrm{w}=45 \mathrm{~mm}, \mathrm{~h}=3 \mathrm{~mm}, \mathrm{t}=1 \mathrm{~mm})$ project the stresses onto the two-dimensional principal stress space between the plane strain tension and simple shear points. The horizontal load cell of the setup provides the data for the shear component of stress, $\tau_{x y}$, and the vertical load cell provides the tensile, $\sigma_{y y}$, data. The horizontal stress component, $\sigma_{x x}$, can not be measured on this facility. However, it is assumed based on basic theories on mechanics of materials that the horizontal stress is always proportional to the vertical stress. This assumption will be used only in the discussion part upon relating the amount of tension to the hydrostatic stress.

The strain is measured real-time on the material surface using a camera and dot-tracking software. 16 black dots are applied to the specimen surface before the test and the corresponding positions are recorded with a frequency of approximately $10 / \mathrm{sec}$. The data is post-processed and averaged to find the strain that accumulates in the material. The approximate resolutions of the strain and stress measurements are $0.05 \%$ and $2 \mathrm{MPa}$, respectively.

\subsection{Magnetic sensor}

The transformation from austenite to martensite is monitored with a magnetic sensor utilizing the permeability difference of the two phases which is in the order of 100 [17]. The sensor proves to supply a steady and representative signal that measures the amount of the martensite phase throughout the experiment. This signal is disturbed by several factors which are removed by a calibration procedure. It is stated in [17] that temperature as well as the stress and strain affect the permeability of martensite due to the magnetostriction phenomenon. In addition to these, the tool steel clamps used in the current tests influence the signal. Therefore, the recorded signal is post-processed to eliminate these interferences. Once a clean signal is attained a correlation with the actual amount of martensite, $\varphi$, is performed by metallographic inspection. An important step before the inspection is the freezing of the microstructure. If unaged the austenite continues to transform isothermally after the tests due to the accumulated plastic strain. Hence, all samples were heat treated at $500{ }^{\circ} \mathrm{C}$ for 30 minutes immediately after the tests. The samples were cut through the length and polished using standard techniques after which the color etchant 
Lichtenegger-Blöch solution was used to reveal the austenite and martensite phases which have adequate amount of contrast to be quantified by a standard image processing tool. One of the images used for correlation is presented in figure 2 .

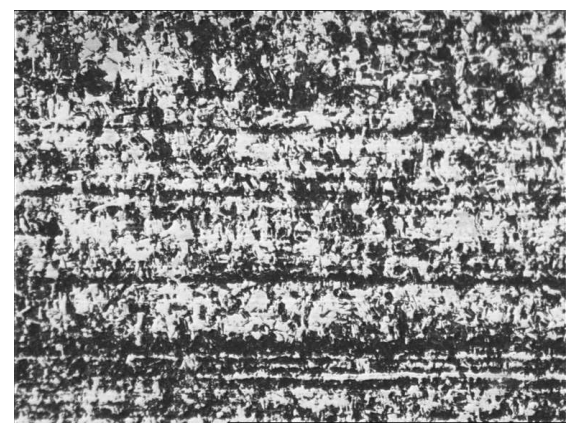

Figure 2: A metallographic sample showing 59\% martensite (dark) and 41\%austenite (light).

\subsection{Material}

The material used in the tests is $12 \mathrm{Cr}-9 \mathrm{Ni}-4 \mathrm{Mo}$ (ASTM A 564) austenitic stainless steel. The nominal composition is given in table 1 . The transformation characteristics of this steel is studied thoroughly in [10][17] by uniaxial tests at different temperatures. The sheet material was fully austenitic in as received condition and the test samples were spark eroded to the exact required shape. Kept at a constant $80^{\circ} \mathrm{C}$ until the tests, no prior isothermal transformation was observed on the samples.

\begin{tabular}{|c|c|c|c|c|c|c|c|}
\hline $\mathrm{C}+\mathrm{N}$ & $\mathrm{Cr}$ & $\mathrm{Ni}$ & $\mathrm{Mo}$ & $\mathrm{Cu}$ & $\mathrm{Ti}$ & $\mathrm{Al}$ & $\mathrm{Si}$ \\
\hline$<0.05$ & 12.0 & 9.1 & 4.0 & 2.0 & 0.9 & 0.4 & $<0.5$ \\
\hline
\end{tabular}

Table 1: Chemical composition of the steel (ASTM A 564) used in the experiments in wt\%.

\section{Results}

\section{$3.1 \quad$ Tests}

In the context of this study two types of tests, namely proportional and non-proportional, were carried out. In the proportional tests the stress state imposed on the material was kept constant during the complete deformation process. This was achieved by keeping the vertical and shear deformation rates in a constant proportion, $D_{y y} / D_{x y}=2 \tan \alpha$, where $\alpha$ is the deformation angle. Whereas in the non-proportional tests the samples were initially sheared, $\alpha_{1}=0$, until $\sim 50 \%$ martensite and then the deformation state was changed to a combination of shear and tension, $\alpha_{2} \neq 0$.

\subsection{Proportional tests}

The range from simple shear to plane strain tension has been covered in 7 steps. The deformation angle $\alpha$ was set to different values with $15^{\circ}$ intervals to cover the space between simple shear, $0^{\circ}$, and plane strain tension, $90^{\circ}$, evenly. The results of these tests are plotted in figure 3 for $\sigma_{y y}-\epsilon_{e q}, \tau_{x y}-\epsilon_{e q}$ and in figure 4 for $\varphi-\epsilon_{e q}$.
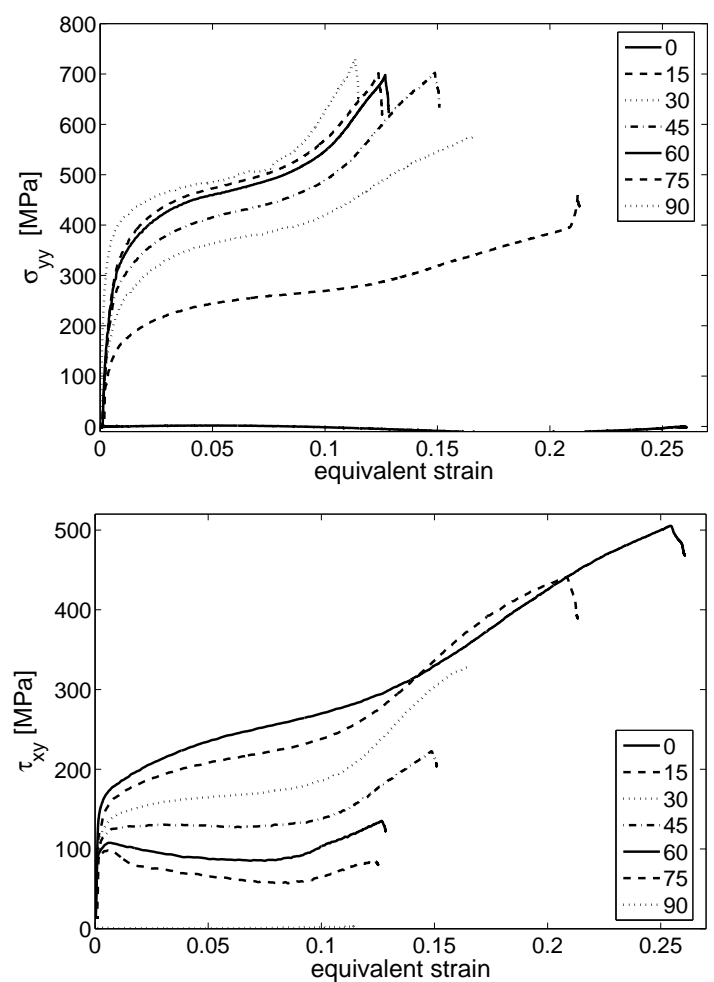

Figure 3 : Stress vs. equivalent strain curves for the proportional tests. Legend denotes the deformation angle, $\alpha$. 


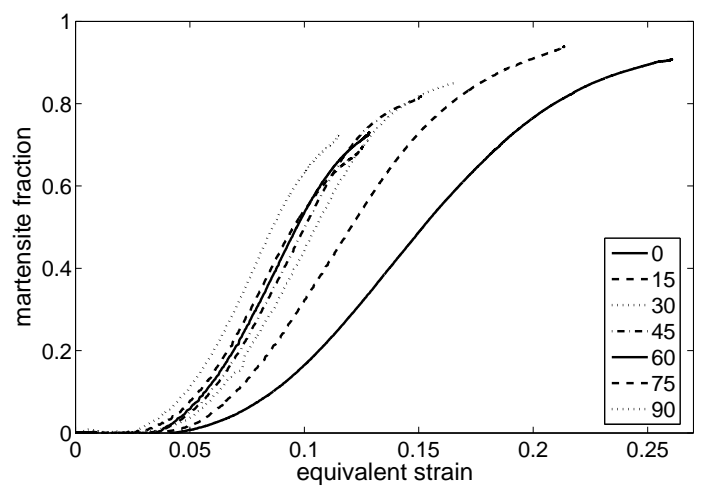

Figure 4: Martensite fraction vs. equivalent strain curves for the proportional tests.

By repeating some of the tests the reproducibility was analyzed and concluded to be excellent. Small deviations were observed only at very high strain levels. The reason of this behavior is twofold: At high tensile deformation the sensor starts to be disturbed from its position in the vertical direction and loses perfect contact with the material. Likewise, at high shear deformation the material starts to wrinkle and this again causes a disturbance in the contact.

A thorough analysis on figure 4 shows that all the transformation curves have the same shape within the margins of experimental error. This behavior is in line with the theory that the transformation is driven by plastic strain whereas the amount of tension influences the rate. This can be more clearly visualized when the amount of strain needed to transform $40 \%$ martensite for each test are plotted against the tensile stress at the corresponding points, as in figure 5. It is observed that among the individual curves there exists a linear relationship that scales with the amount of tensile stress.

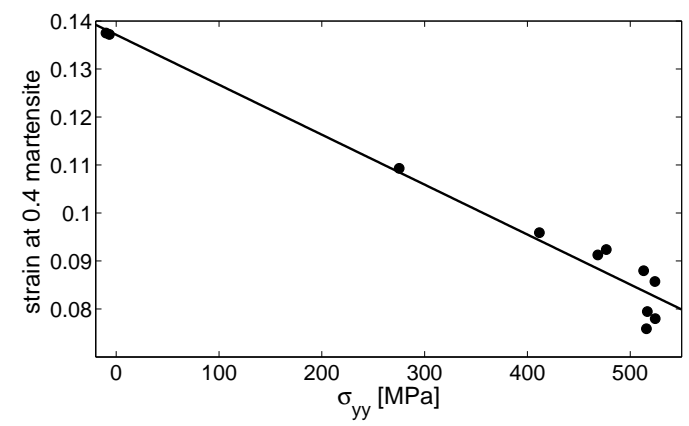

Figure 5 : Equivalent strain at $40 \%$ martensite vs. corresponding tensile stress.

Due to the reasons introduced in section 2.2 it is not possible to relate this phenomenon quantitatively to the hydrostatic stress instead of tension. However, based on the assumption that the material obeys the isotropic J2 plasticity model in the absence of martensitic transformation, the hydrostatic stress will always be proportional to the tensile stress in this setup. Hence, the increase in transformation speed might be concluded to originate from the increase in the mechanical driving force with the hydrostatic stress. A volumetric expansion accompanies the martensite formation therefore, the hydrostatic stress will act on every variant to generate an isotropic increase in the driving force which, according to the formulation by $\mathrm{Pa}$ tel \& Cohen [6], increases linearly with hydrostatic stress.

\section{$3.3 \quad$ Non-proportional tests}

The range from simple forward shear, $0^{\circ}$, to reverse shear, $180^{\circ}$, was also covered in 7 steps. In the $\alpha_{2} \neq 0$ stage, the same procedure as the proportional tests was followed. The transition from $\alpha_{1}=0$ was performed continuously without an elastic unloading step in between. The results, $\varphi-\epsilon_{e q}$, are plotted in figure 6 . The aim of the non-proportional tests was to observe the transformation behavior upon a sudden change in stress state. The results show that there is no significant phenomenon associated with this except that the transformation proceeds continuously while the rate changes from the rate at $\alpha_{1}=0$ to the corresponding proportional deformation rate.

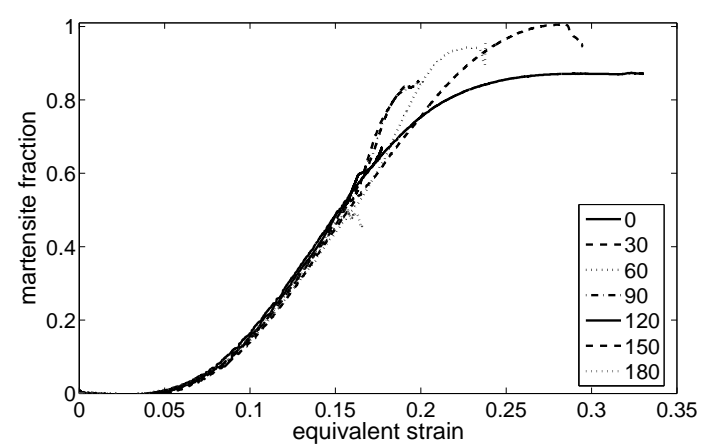

Figure 6 : Martensite fraction vs. equivalent strain curves for the non-proportional tests.

\subsection{Discussion}

The linear relation between the rate of transformation and tensile stress can be utilized in 
a numerical model to predict the transformation curve in a non-proportional test. In figure 7 the non-proportional test with $\alpha_{2}=90$ is reproduced using the simple shear transformation curve and the recorded tensile stress. It is observed that the smooth increase in transformation rate upon the change of stress state is very well captured.

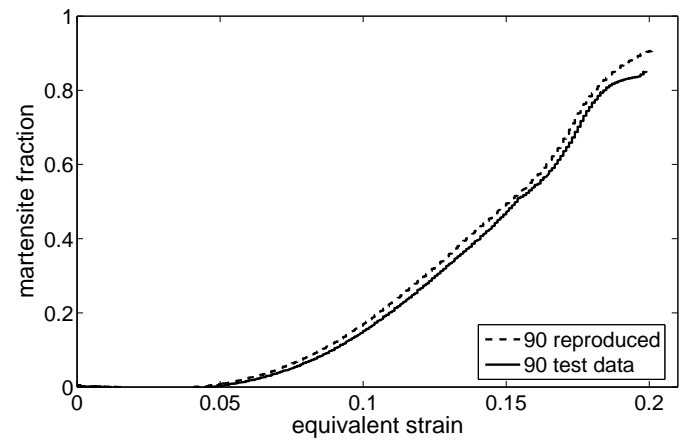

Figure 7: The test results of the non-proportional test with $\alpha_{2}=90$ and the reproduced results using the simple shear curve.

\section{Conclusion}

Strain induced martensitic transformation as a function of stress state and strain path has been studied on a metastable austenitic stainless steel using a biaxial test equipment. The results provide quantitative relations in the understanding of the mechanisms of martensitic transformation aided by deformation.

A constant stress state was imposed on the material during the proportional tests and the influence of the tensile stress on transformation kinetics was investigated. A linear relation between the amount of tension and the transformation rate was found which is in accord with the Patel \& Cohen theory on the action of applied stress on martensitic transformations. Based on this theory it can be concluded that an isotropic increase in mechanical driving force shows itself in a proportional increase in the rate of transformation.

In the non-proportional tests the stress state on the material was switched suddenly from simple shear to a combination of shear-tension after $\sim 50 \%$ transformation. No specific occurrence related to this change was observed. The transformation rate however was found to follow the linear relation that has been formulated using the proportional tests.

\section{Acknowledgement}

This research was carried out under project number 02EMM30-2 in the framework of the FOM-NIMR research programme in the Netherlands.

\section{References}

[1] T. Angel, Journal of the iron and steel institute 177 (1954) 165-174

[2] G.B. Olson, M. Cohen, Metallurgical Transactions 6A (1975) 791-795

[3] A.C.E. Reid, G.B. Olson, Materials Science and Engineering A 309-310 (2001) 370-376

[4] V.I. Levitas, A.V. Idesman, G.B. Olson, Acta Materialia 47 (1999) 219-233

[5] M. Wechsler, D. Lieberman, T. Read, Transactions of the AIME 197 (1953) 15031515

[6] J.R. Patel, M. Cohen, Acta Metallurgica 1 (1953) 531-538

[7] M. Cherkaoui, M. Berveiller, X. Lemoine, International Journal of Plasticity 16 (2000) 1215-1241

[8] F. Marketz, F.D. Fischer, Computational Materials Science 3 (1994) 307-325

[9] R.G. Stringfellow, D.M. Parks, G.B. Olson, Acta Metallurgica et Materialia 40 (1992) 1703-1716

[10] J. Post, J. Huétink, H.J.M. Geijselaers, R.M.J. Voncken, Journal de Physique IV 112 (2003) 417-420

[11] H.N. Han, C.G. Lee, C-S. Oh, T-H. Lee, S-J. Kim, Acta Materialia 52 (2004) 52035214

[12] Y.H. Yan, G.Y. Kai, M.D. Jian, Materials science and Engineering A 441 (2006) 331335

[13] A.A. Lebedev, V.V. Kosarchuk, International Journal of Plasticity 16 (2000) 749767 
[14] Q. Furnémont, F. Delannay, P.J. Jacques, Journal de Pyhsique IV 112 (2003) 421-424

[15] J.C. Videau, G. Cailletaud, A. Pineau, Journal de Physique IV 6 (1996) 465-474

[16] M. Coret, S. Calloch, A. Combescure, Eu- ropean Journal of Mechanics A/Solids 23 (2004) 823-842

[17] J. Post, On the constitutive behavior of Sandvik Nanoflex, PhD Thesis, University of Twente, 2004 